\title{
O engajamento discente durante a pandemia por Covid-19 frente ao ensino remoto e
}

\section{ao uso do GoogleClassroom}

The student engagement during the Covid-19 pandemic against remote teaching and the use of GoogleClassroom

Participación de los estudiantes durante la pandemia del Covid-19 hacia el aprendizaje remoto y el uso de GoogleClassroom

\section{Resumo}

A crise sanitária provocada pelo Covid-19 impulsionou as aulas a distância através do ensino remoto e o uso de plataformas como o GoogleClassroom desenvolvido com foco na integração entre professores e alunos. O presente artigo teve como objetivo avaliar o engajamento discente durante a pandemia por Covid-19 frente ao ensino remoto e ao uso do GoogleClassroom. Tratou-se de um estudo de natureza básica, por meio de revisão bibliográfica, de caráter descritivo e exploratório, cujos dados foram tratados de maneira qualitativa para compreender a participação dos alunos nas aulas síncronas utilizando a ferramenta GoogleClassroom como plataforma de acompanhamento dos conteúdos abordados em aulas virtuais durante a pandemia. A pesquisa foi realizada por meio da realização de buscas nos repositórios bases Periódicos CAPES e Google Scholar, utilizando os seguintes descritores: "engajamento", "ensino remoto", "pandemia por Covid-19" e "GoogleClassroom". Após a seleção criteriosa, foram selecionados 15 artigos publicados em periódicos nacionais dos últimos 5 anos. A análise dos artigos demonstra que as ferramentas educacionais de interação online permitiram a realização de um trabalho inovador, focado na formação inicial e continuada de alunos, bem como na integração entre os participantes da turma. $\mathrm{O}$ uso de plataformas online e aulas virtuais favoreceram o planejamento de ações, proporcionando ganho de conhecimento individual e coletivo.

Palavras-chave: GoogleClassroom; Engajamento; Ensino remoto; Pandemia.

\begin{abstract}
The health crisis caused by Covid-19 boosted distance classes through remote learning and the use of platforms such as Google Classroom developed with a focus on integration between teachers and students. This article aimed to assess student engagement during the Covid-19 pandemic towards remote learning and the use of Google Classroom. This was a study of a basic nature, through bibliographical review, descriptive and exploratory, whose data were treated in a qualitative way to understand the participation of students in synchronous classes using the Google Classroom tool as a platform for monitoring the contents covered. in virtual classes during the pandemic. The research was carried out by conducting research in the CAPES and Google Scholar journals databases, using the following descriptors: "engagement", "remote teaching", "pandemic by Covid-19" and "Google Classroom". After careful selection, 15 articles published in national journals between the years 2020 and 2021 were selected. The analysis of the articles demonstrates that the educational tools for online interaction allowed the realization of an innovative work, focused on
\end{abstract}


the initial and continuing education of students, as well as in the integration among the participants of the class. The use of online platforms and virtual classes favored action planning, providing individual and collective knowledge gain.

Keywords: Googleclassroom; Engagement; Remote teaching; Pandemics.

\begin{abstract}
Resumen
La crisis de salud provocada por Covid-19 impulsó las clases a distancia a través del aprendizaje remoto y el uso de plataformas como GoogleClassroom desarrolladas con un enfoque en la integración entre profesores y alumnos. Este artículo tuvo como objetivo evaluar la participación de los estudiantes durante la pandemia Covid-19 hacia el aprendizaje remoto y el uso de GoogleClassroom. Se trató de un estudio básico, mediante revisión bibliográfica, descriptiva y exploratoria, cuyos datos fueron tratados de forma cualitativa para comprender la participación de los estudiantes en clases sincrónicas utilizando la herramienta GoogleClassroom como plataforma de seguimiento de los contenidos cubiertos en las clases virtuales durante la pandemia. La investigación se llevó a cabo mediante la investigación en las bases de datos de las revistas CAPES y Google Scholar, utilizando los siguientes descriptores: "engagement", "enseñanza remota", "pandemia por Covid-19" y "GoogleClassroom". Luego de una cuidadosa selección, se seleccionaron 15 artículos publicados en revistas nacionales entre los años 2020 y 2021. El análisis de los artículos demuestra que las herramientas educativas para la interacción en línea permitieron la realización de un trabajo innovador, enfocado a la formación inicial y continua de los estudiantes, así como en la integración entre los participantes de la clase. El uso de plataformas en línea y clases virtuales favoreció la planificación de acciones, proporcionando un conocimiento individual y colectivo.
\end{abstract}

Palabras clave: GoogleClassroom; Engagement; Remote learning; Pandemia.

\title{
1. Introdução
}

Uma pandemia foi anunciada, o vírus viajou o mundo, se instalou nos corpos, milhares de pessoas ficaram gravemente doentes, o sistema de saúde de países ricos e pobres entrou em colapso, as mortes se multiplicaram, o pânico se instalou, as tão comuns relações comerciais e pessoais foram comprometidas e o direito de ir e vir foi impactado. O comércio, as escolas, as práticas esportivas, as atividades culturais, os encontros, os contatos, as conversas e os afetos foram interrompidos (Couto et al., 2020).

Os aeroportos foram fechados, em alguns lugares os transportes públicos pararam, as viagens e os passeios foram suspensos. As atividades escolares tiveram que ser bruscamente interrompidas. Uma condição das pestes do passado voltou à cena: viver em distanciamento social, perdidos em meios a informações contraditórias e incompletas (Harari, 2020).

Diante do contexto de pluralidade territorial presente no Brasil é comum que ocorram disparidades culturais e educacionais. A legislação educacional ao se preocupar em manter uma qualidade de ensino e de garantir preceitos básicos aos estudantes desenvolveu a Base Nacional Comum Curricular (BNCC). A BNCC se define como um conjunto de normas elaboradas em 2015, com versões subsequentes que, integradas, garantem as aprendizagens essenciais que cada aluno deve ter nas escolas, orientando a elaboração de um currículo independente do município e o estado em que o aluno se encontra e trazendo um contexto uniformizado ao ensino (Instituto Ayrton Senna, 2021).

A pandemia iniciada em 2020 veio de forma repentina e com vias de impedimento a algumas das 10 diretrizes nas quais a BNCC se norteia, trazendo grandes desafios. O enfrentamento desses desafios para o ensino e de outros que despontam a partir da Covid-19, permitiu questionar se num futuro próximo o ensino poderia ser planejado considerando também o uso de tecnologias baseadas na internet de forma síncrona e assíncrona.

Diante deste contexto, o presente artigo avaliou o uso das tecnologias digitais como recurso para mediação do processo de engajamento dos discentes em tempos de pandemia discutindo às potencialidades pedagógicas para o ensino remoto com uso da plataforma virtual Google Classroom.

\section{Metodologia}

Esse estudo trata-se de uma revisão bibliográfica, de caráter descritivo e exploratório, cujos dados foram tratados de maneira qualitativa para compreender a participação dos alunos nas aulas síncronas utilizando a ferramenta GoogleClassroom 
como plataforma de acompanhamento dos conteúdos abordados em aulas virtuais durante a pandemia. A pesquisa foi realizada por meio de buscas nos repositórios bases Periódicos CAPES e Google Scholar, utilizando os seguintes descritores: "engajamento", "ensino remoto", "pandemia por Covid-19" e "GoogleClassroom". Foram selecionados 15 artigos publicados em periódicos nacionais dos últimos 5 anos. A seleção dos artigos ocorreu com base na análise prévia do título e resumo, e posteriormente, análise detalhada de todo trabalho.

\section{Contextualização}

A pandemia da COVID-19 remeteu à reflexão sobre o quanto é importante a relação interpessoal, pois a falta de contato físico vivenciada por muitos evidenciou a dificuldade para expressar sentimentos e de uma comunicação nos ambientes escolares. No entanto, em um mundo assolado por uma pandemia, a tecnologia foi a ferramenta que contribuiu para o contato profissional, sentimental e/ou familiar.

Com as regras impostas para o distanciamento social foi revelada a importância de se relacionar fisicamente, um com os outros, e diante da crise sanitário foi percebida uma nova cultura mundial. A preocupação se voltou para além da assistência a pacientes com suspeita ou confirmação de Covid-19, com objetivos de cura dos já infectados pelo vírus, mas, também, para a democratização do acesso ao ensino. De fato, estratégias para o mundo pós-pandemia se tornam uma demanda mundial e cada dia vem surgindo para sanar as consequências vindouras dos limites impostos.

Na preocupação de ser um fator essencial para ajudar na contínua e necessária busca do aprendizado, buscou-se formas de continuar o processo de ensino/aprendizagem. O ensino remoto é uma estratégia didática e pedagógica criada para diminuir os impactos das medidas de isolamento social sobre a aprendizagem e, por meio das metodologias ativas, propõe um instrumento tecnológico para reuniões no formato de vídeo conferência (Bacich et al., 2015; Almeida, 2003).

Percebe-se que o processo de ensino e aprendizagem no ambiente remoto deve envolver provocação, desenvolvimento de habilidades críticas e competências, colhendo elementos que permitam avaliar a proposta pedagógica. Ademais, como destacam de Carvalho Borba et al. (2014), o uso de tecnologias digitais pode contribuir para a compreensão de professores e pesquisadores no tocante às transformações que ocorrem na aprendizagem dos alunos.

Segundo Abramowicz e Tebet (2017), há um grande desafio inerente à BNCC que seria em como a proposta de unificação da educação enfrentaria diversos problemas. Há uma ótica do que pode ser perdido nesse processo e em como unificar as diferenças construindo um bem comum a todos. Os autores trazem uma comparação do projeto ao papel de Estado, que precisa legislar sobre diferentes culturas da forma mais justa e igualitária possível, garantindo todos os direitos a cada cidadão. Nesse contexto, o papel do educador se torna mais complexo para garantir esses valores supracitados, levantando debates acerca desse tema em todo o país, e daí o processo de ensino remoto vem como via a ser avaliada e aplicada da melhor maneira nos mais diferentes grupos sociais.

Santo e Trindade (2020) demarcam o surgimento do ensino remoto emergencial (ERE) a partir de situações atípicas como pandemias e outras catástrofes e o define como possibilidade para a continuidade das atividades pedagógicas com o objetivo de diminuir os prejuízos derivados da suspensão das aulas presenciais.

\section{O Ensino Remoto}

Diante da pandemia causada pelo coronavírus da síndrome respiratória aguda grave 2 (SARS-CoV-2), o Ministério da Educação (MEC) brasileiro atendendo à solicitação da Associação Brasileira de Mantenedoras de Ensino Superior (ABMES) e sob orientações do Conselho Nacional de Educação (CNE) publicou a portaria n 343, de 17 de março de 2020, que regulamenta as Instituições de Ensino a substituírem aulas presenciais por aulas em meios digitais enquanto durar a situação de pandemia do Novo Coronavírus - COVID-19 (Brasil, 2020). 
As Instituições de Ensino em atendimento à portaria do MEC fecharam seus campus temporariamente e passaram a vislumbrar nas Tecnologias de Informação e Comunicação (TICs) possibilidades de continuar os processos de ensino e aprendizagem remotamente envolvendo alunos e professores por meio dos recursos tecnológicos oferecidos pela internet (Brasil, 2020).

A inclusão dos termos emergencial e remoto na definição das práticas de ensino, desenvolvidas no contexto que o mundo está vivendo desde 2020 é fundamental na perspectiva de Tomazinho (2020), pois o que caracteriza o remoto é a impossibilidade de professores e estudantes frequentarem as escolas em razão da tentativa de contenção da propagação do novo coronavírus. O emergencial situa a temporalidade desta alternativa, uma vez que os planejamentos pedagógicos de todas as instituições de ensino foram interrompidos abruptamente, com riscos de não mais serem aproveitados no ano de 2020 e novas alternativas precisaram ser adotadas na mesma velocidade.

Desta forma, o ensino remoto foi uma alternativa emergencial e pontual adotada por instituições de ensino para possibilitar que o vínculo pedagógico não fosse rompido totalmente. Tem sido desenvolvido no Brasil das mais diversas formas, com a mediação de tecnologias digitais. Nomear as modalidades, tipologias e práticas de ensino é importante para evitar o enfraquecimento e fragilização das áreas educacionais.

Em uma perspectiva de oportunizar o engajamento dos estudantes de forma flexível e virtual, conforme citada por Daudt (2015), acredita-se ser possível continuar desenvolvendo o processo educacional, com o apoio das tecnologias, diminuindo os impactos ou efeitos do isolamento social na formação de milhares de alunos afastados da estrutura física da sala e da vivência escolar.

\section{As Plataformas Virtuais e o Googleclassroom}

Durante a pandemia observou-se o crescimento do uso das plataformas digitais, tanto as utilizadas majoritariamente para entretenimento, como o Facebook ou o Instagram, quanto aquelas com fins educacionais, como Teams (Microsoft), GoogleClassroom, Google Meet, Zoom, RNP, dentre outras. Estas plataformas permitem interações on-line como: conversar, compartilhar, comentar, pesquisar, fazer compras, ouvir música, assistir vídeos etc., permitindo o delineamento sobre a maneira como se vive e como a sociedade é organizada.

Estruturadas por dados e organizadas por meio de algoritmos, as plataformas são projetadas, fundamentalmente, para organizar interações entre usuários (Van Dijck, et al., 2018) controlando os seus dados e direcionando esses sujeitos a uma rede infinita de informação a partir de um descritor de busca inicial. Por exemplo, ao procurar em um site por um produto, os sujeitos passam a receber distintas informações sobre esse item e/ou afins.

O GoogleClassroom surge como uma proposta para hospedar aulas assíncronas, substituindo os encontros presenciais temporariamente e tem como objetivo tornar a aprendizagem mais significativa no período de isolamento social. Essa plataforma permite total autonomia para o professor personalizar o ambiente virtual e a configurar as postagens para que fique de acordo com seu planejamento didático (Conforto \& Vieira, 2015).

Percebe-se que, por se tratar de uma ferramenta acessível, gratuita e de fácil usabilidade, principalmente pelas pessoas que já convivem em diversos ambientes permeados de ferramentas digitais, o Google Classroom teve fácil aceitação por parte do professor, pois facilita suas atividades diárias, como a função de programar as postagens e de hospedar todas as atividades das turmas em um só lugar. Dessa forma, o professor pode corrigir/analisar as atividades de forma online, em qualquer horário e lugar, pelos diferentes dispositivos digitais.

Um fator que leva o GoogleClassroom a estar entre os aplicativos favoritos dos alunos é que, por se tratar de uma ferramenta Google, ele não ocupa a memória dos dispositivos, pois mantém os materiais (artigos, livros, vídeos e demais) compartilhados pelo professor em nuvem. 
Segundo Daudt (2015), as videoconferências se tornaram populares entre os alunos que a todo o momento procuram um pouco de calor humano durante a quarentena. Numa perspectiva mais crítica acerca das tecnologias apresentadas neste estudo, entende-se que a inserção estratégica dos recursos supracitados não ocorre em um processo linear. Com base nisso, as plataformas virtuais apresentam um aspecto de inovação para as aulas e para os processos de ensino e aprendizagem por promoverem o protagonismo do estudante e seu engajamento com o objeto de estudo, o que agrega ainda mais valor e significância aos conhecimentos apreendidos. Os jovens de hoje são chamados nativos digitais e as plataformas virtuais podem ser ferramentas de aprendizagem alinhadas a esta geração.

\section{Impacto na Educação com a Pandemia por Covid-19}

A fim de avaliar o impacto da COVID-19 nos alunos de diferentes faixas etárias, Chaturvedi et al., (2021) levantou os seguintes questionamentos: tempo gasto em aulas síncronas e assíncronas, meios usados para aprendizagem e as ferramentas que os mesmos utilizaram, hábitos de sono, rotina de exercícios diários e os efeitos subsequentes no peso e saúde mental. No estudo desenvolvido foram entrevistados pelas plataformas digitais 1.182 estudantes entre 07 e 59 anos, de vários institutos educacionais em Delhi - National Capital Region (NCR), Índia.

A pesquisa identificou que, para lidar com o estresse e a ansiedade, os participantes adotaram diferentes mecanismos de enfrentamento e também buscaram a ajuda de seus entes próximos. A pesquisa também examinou o envolvimento do aluno em plataformas de mídia social entre diferentes categorias de idade.

Neste estudo, os autores sugerem que as autoridades públicas devem tomar todas as medidas necessárias para melhorar a experiência de aprendizagem, mitigando os impactos negativos causados pelo surto da COVID -19, dentre elas foram citadas: suporte emocional aos estudantes, treinamento adequado de educadores para as habilidades digitai e investimento na educação online para aprimorar a experiência de aprendizagem.

Os dados apontados pela pesquisa retratam as realidades encontradas nas mais diversas unidades de Ensino ao redor do mundo. Em âmbito nacional, os estudantes da rede pública de ensino sofreram um apagão educacional por praticamente um ano, e durante a implantação da modalidade remota, a falta de acesso aos recursos tecnológicos agigantou ainda mais o abismo que separa os estudantes de rede pública e privada no Brasil.

\section{O Tempo de Aula e a Atenção do Estudante}

Um ponto importante a se destacar para essa modalidade de ensino está relacionado ao tempo "ideal" de aulas, ou seja, tempo aproximado onde se consegue prender a atenção do público-alvo. Esse tema vem sendo discutido ao longo dos anos por muitos professores, psicólogos e neurocientistas, sendo que, a cada década, muitas pesquisas defendem que o estudante já não consegue se concentrar por tanto tempo em sala de aula, e com isso, o tempo de hora/aula remota veio sofrendo reduções.

Segundo Mora (2017), o educador deve pensar em ciclos de aprendizagem, mesclando os formatos de ensino a cada 15 minutos (discurso, leitura, atividade prática, dinâmicas em grupo, etc.), para que os alunos não fiquem entediados e tenham um poder de concentração maior. Lembrando que, conforme os conceitos andragógicos, cada indivíduo aprende de uma maneira, portanto, o educador deve estar preparado para a diversidade das turmas e utilizar as metodologias ativas direcionadas ao público em sala.

O "poder de atenção" dos indivíduos é cada vez menor, e paralelo a isso, o engajamento, as formações, o preparo, o uso de tecnologias, e outros fatores precisam fundamentalmente fazer parte da prática pedagógica. Os professores cada vez mais precisarão transformar a aula em um momento precioso de interação onde o aluno é ouvido e motivado enquanto aprende de forma prática, contextualizada e natural. 


\section{As Aulas Remotas e o Engajamento dos Estudantes}

Numa situação de aula remota, no que tange especificamente à etapa de utilização de plataformas virtuais como ferramenta para mediação do ensino-aprendizagem assume o papel de atividade de problematização para o tema de aula proposto e que seja apresentado como uma ferramenta de estímulo e engajamento aos alunos.

Vale ressaltar que as plataformas virtuais têm vários aspectos positivos enquanto ferramenta para o processo de ensino e aprendizagem em diversas áreas do conhecimento. Em primeiro lugar, trata-se claramente de mais uma ferramenta a ser considerada no planejamento do professor e tem uma premissa desafiadora que cria engajamento do aluno e envolvimento com múltiplos conteúdos, e também os relacionando a outras disciplinas, tornando a aprendizagem mais significativa, promovendo o resgate de uma série de conceitos técnicos de forma lúdica e divertida.

Em relação ao engajamento dos estudantes os estudos identificaram que este ocorre em três níveis: 1 - o engajamento comportamental associado a noção de participação; 2 - o engajamento emocional relaciona-se às reações positivas e negativas do estudante aos fatores e recursos escolares e 3 - o engajamento cognitivo está relacionado a ideia de investimento, fazer esforço para entender ideias complexas e deter habilidades difíceis (Fredricks et al., 2004).

Desta forma, todas as alterações no ambiente de ensino são feitas baseadas em duas convicções, ambas sustentadas em resultados de diversas pesquisas: a primeira é que o professor não pode instruir, mas pode proporcionar aos alunos experiências com boas oportunidades de aprendizagem alterando o ambiente de aprendizagem, o contexto em que a aprendizagem se dá (Moreira \& Borges, 2005 \& Moreira, 2003 \& Schön, 1987). Na segunda, o engajamento do aluno é a chave para garantir a aprendizagem que faz a diferença (Campbell, et al., 1994). Essa última noção pode ser utilizada para parafrasear o famoso dito de Ausubel - “o fator singular mais importante a influenciar a aprendizagem é aquilo que o aprendiz já sabe. Descubra isto e ensine de acordo" (Ausubel, 1968). "O fator singular mais importante a influenciar a aprendizagem é o engajamento ativo do aprendiz com o material. Obtenha isto e ensine por quaisquer métodos que retenham este engajamento" (Campbell, et al., 1994).

Nesse contexto, é necessário transitar neste ensino remoto de emergência, importante numa primeira fase para uma educação digital de qualidade em rede. Mais do que a transferência de práticas presenciais, é necessário utilizar modelos de aprendizagem virtuais que incorporem processos de desconstrução e que promovam ambientes de aprendizagem colaborativos e construtivos, tendo em mente que aulas remotas não devem ser confundidas com a EaD tradicional, onde o aluno precisa desenvolver habilidades autodidatas para ter um bom desempenho (Moreira, et al., 2020).

A questão do engajamento dos alunos perpassa pelas diferenças e desigualdades sociais que acabam por excluir parte dos alunos do acesso ao conhecimento através das mídias digitais, sendo imensamente prejudicados em seu processo de aquisição de informações. Sobre esta questão, têm-se observado debates nas mídias que revelam as desigualdades no acesso à educação de qualidade, como foi veiculado pelo portal eletrônico "Brasil de Fato - Uma visão popular do Brasil e do Mundo", no dia 04 de junho de 2020, pela jornalista Caroline Oliveira em reportagem com João Carlos Salles, presidente da Associação Nacional dos Dirigentes das Instituições Federais de Ensino Superior (Andifes) e Reitor da Universidade Federal da Bahia. A pandemia expôs as condições desiguais em que os estudantes brasileiros se encontram. Segundo a jornalista Caroline, para Salles:

No início da implementação das medidas de isolamento social, quando as aulas passaram a ser remotas houve quem imaginasse que as tecnologias digitais poderiam dar continuidade integral, e com a mesma qualidade às aulas presenciais. "Isso é evidentemente falso. Segundo uma pesquisa feita pelo Comitê Gestor da Internet no Brasil (CGI.br), em 2018, 58\% dos domicílios no Brasil não têm computadores e 33\% não possuem internet. A desigualdade é muito forte. Desigualdade de recursos, de condição para estudar, de tempo, dedicação. Tudo isso, é claro, afeta a educação" (Salles, 2020).

Ainda para Salles, Reitor da Universidade Federal da Bahia: 
O Estado fracassou em incluir efetivamente em grandes camadas da população, estudantes desde a educação básica até a educação superior e em preparar as habitações, ou seja, as políticas públicas que deem a mínima tranquilidade para que as pessoas possam, em situação extrema como é a que estamos vivendo, continuar a sua formação, os seus estudos (Salles, 2020).

Portanto, confirma-se a afirmação dos gestores quando se referem ao ensino remoto e uso das plataformas, pois os maiores desafios das escolas neste momento é o engajamento dos estudantes nas atividades remotas devido a fatores como acesso a equipamentos, conexão com internet e outros fatores econômicos e sociais que geram exclusão de acesso à educação de qualidade para todos os alunos. Nesse contexto, conceitos de metodologias ativas aplicadas (Buzz \& Mackedanz, 2017) se tornam imprescindíveis ao educador, que deve ter em mente os pontos abordados no que tange ao ensino a distância e ao mesmo tempo manter a instigação no aluno pelo conhecimento passado em sala.

\section{Considerações Finais}

Diante das análises realizadas, nota-se que o cenário educacional que sempre foi desafiador, torna-se ainda mais, nesse contexto pandêmico, onde o distanciamento social e consequente fechamento das escolas foram essenciais para que vidas fossem poupadas, mas que trouxe inúmeros efeitos no que tange as práticas de ensino e aprendizagem. Os professores vêm tendo a oportunidade de compartilhar conteúdos através de plataformas online como o Google Classroom e ensinar por aulas remotas, mas alguns estudantes ainda encontram dificuldades no engajamento diante da falta de acesso à internet e outros motivos pontuais.

\section{Referências}

Abramowicz, A., \& Tebet, G. G. D. C. (2017). Educação Infantil: um balanço a partir do campo das diferenças1. Pro-posições, 28, 182-203. 10.1590/19806248-2016-0114

Almeida, M. E. B. D. (2003). Educação a distância na internet: abordagens e contribuições dos ambientes digitais de aprendizagem. Educação e pesquisa, 29, 327-340. 10.1590/S1517-97022003000200010.

Ausubel, D. P., Novak, J. D., \& Hanesian, H. (1968). Educational psychology: A cognitive view. Holt, Rinehart and Winston.

Bacich, L., Neto, A. T., \& de Mello Trevisani, F. (2015). Ensino híbrido: personalização e tecnologia na educação. Penso Editora.

de Carvalho Borba, M., da Silva, R. S. R., \& Gadanidis, G. (2020). Fases das tecnologias digitais em Educação Matemática: sala de aula e internet em movimento. Autêntica Editora.

Brasil. (2020) Parâmetros Curriculares Nacionais: Ensino Médio. Brasília: Ministério da Educação, http://basenacionalcomum.mec.gov.br/abase/

da Silva Buss, C., \& Mackedanz, L. F. (2017). O ensino através de projetos como metodologia ativa de ensino e de aprendizagem. Revista Thema, 14(3), 122131. 10.15536/thema.14.2017.122-131.481.

Campbell, B., Lazonby, J., Millar, R., Nicolson, P., Ramsden, J., \& Waddington, D. (1994). Science: The Salters' approach-a case study of the process of large scale curriculum development. Science education, 78(5), 415-447. https://doi.org/10.1002/sce.3730780503

Chaturvedi, K., Vishwakarma, D. K., \& Singh, N. (2021). COVID-19 and its impact on education, social life and mental health of students: A survey. Children and youth services review, 121, 105866. 10.1016/j.childyouth.2020.105866

Conforto, D., \& Vieira, M. C. (2015). Smartphone na escola: Da discussão disciplinar para a pedagógica. Latin American Journal Of Computing LAJC, 2, 4354. https://lajc.epn.edu.ec/index.php/LAJC/article/view/95/56

Couto, E. S., Couto, E. S., \& Cruz, I. D. M. P. (2020). \# fiqueemcasa: educação na pandemia da COVID-19. Interfaces Científicas-Educação, 8(3), 200-217. $10.17564 / 2316-3828.2020 v 8 n 3 p 200-217$.

Daudt, L. (2015). Ferramentas do google sala de aula que vão incrementar sua aula. https://www.qinetwork.com.br/6-ferramentas-do-googlesalade-aula-quevao-incrementar-sua-aula/.

Frederick, C., \& Biddiss, M. (2005). Grandes pestes de la Historia. El Ateneo.

Fredricks, J. A., Blumenfeld, P. C., \& Paris, A. H. (2004). School engagement: Potential of the concept, state of the evidence. Review of educational research, 74(1), 59-109. 10.3102/00346543074001059.

Harari, Y. N. (2020). Na batalha contra o coronavírus, faltam líderes à humanidade. Companhia das Letras. Editora Schwarz. 
Research, Society and Development, v. 10, n. 11, e130101119451, 2021

(CC BY 4.0) | ISSN 2525-3409 | DOI: http://dx.doi.org/10.33448/rsd-v10i11.19451

Instituto Ayrton Senna (2021). O que é a BNCC. https://institutoayrtonsenna.org.br/pt-br/BNCC/o-que-e-BNCC.html\#bncc.

Mora, Francisco. Francisco Mora (2017): “É preciso acabar com o formato das aulas de 50 minutos”. Entrevista concedida a Ana Torres Menárguez. El País, Brasil, fevereiro. https://brasil.elpais.com/brasil/2017/02/17/economia/1487331225_284546.html.

Moreira, A. F., \& Borges, O. (2006). Por dentro de uma sala de aula de física. Educação e Pesquisa, 32, 157-174. 10.1590/S1517-97022006000100010

Moreira, A. F. (2003). Práticas de interpretação em ambientes de aprendizagem de Física. https://repositorio.ufmg.br/bitstream/1843/EJRA5SFJ2R/1/tese_adelson.pdf

Moreira, J. A., Henriques, S., \& Barros, D. M. V. (2020). Transitando de um ensino remoto emergencial para uma educação digital em rede, em tempos de pandemia. Dialogia, 351-364. 10.5585/dialogia.n34.17123

Oliveira, C. (2020). Portal eletrônico Brasil de Fato: "Uma visão popular do Brasil e do Mundo". https://www.brasildefato.com.br/2020/06/04/com-aulasremotas-pandemia-escancara-desigualdade-no-acesso-a-educacao-de-qualidade.

Santo, E. D. E., \& Dias-Trindade, S. (2020). Educação a Distância e Educação Remota Emergencial: convergências e divergências. Machado, Dinamara P. Educação em tempos de COVID-19: reflexões e narrativas de pais e professores. Curitiba: Editora Dialética e Realidade.

Schön, D. A. (1987). Educating the reflective practitioner: Toward a new design for teaching and learning in the professions. Oxford University Press.

Tomazinho, P. (2020). Ensino Remoto Emergencial: a oportunidade da escola criar, experimentar, inovar e se reinventar. Revista do SINEPE/RS, https://medium.com/@paulotomazinho/ ensino-remoto-emergencial-a-oportunidade-da-escola-criar-experimentar-inovar-e-se-reinventar6667ba55dacc.

Van Dijck, J., Poell, T., \& De Waal, M. (2018). The platform society: Public values in a connective world. Oxford University Press. 